\title{
MICRO-CLUSTER Y NUEVO TURISTA
}

\section{MICRO-CLUSTER ET NOUVEAU TOURISTE}

\section{MICRO-CLUSTER AND NEW TOURIST}

\section{AUTORA}

Amélie Fiorello: Universidad Nice Sophia-Antipolis. Niza (Francia). ameliefiorello@hotmail.fr

\section{RÉSUMÉ}

Si le tourisme peut être un important moteur du développement économique et social d'une destination, il peut aussi s'avérer destructeur. Aujourd'hui on assiste à un changement des mentalités et même si le tourisme de masse est loin de disparaître, un nouveau type de consommateur de produits touristiques fait son apparition. Le nouveau touriste est demandeur d'expériences uniques, de contact avec les populations des pays qu'il visite tout en se montrant soucieux de leur bienêtre et de préserver l'environnement naturel qui l'entoure. L'écotourisme communautaire apparaît ainsi comme un projet de tourisme alternatif qui combine les différents aspects du développement durable. Ce type de tourisme peut s'adapter indifféremment aux communautés rurales de pays en voie de développement qu'aux communautés rurales de zones périphériques dans les pays développés. De tels projets peuvent être optimisés s'ils sont développés à travers des clusters de petites tailles ou micro-clusters. 


\section{MOTS CLÉS}

Développement durable - Durabilité économique - Tourisme.

\section{RESUMEN}

Si bien el turismo puede ser un motor importante de desarrollo económico y social de un destino, también puede ser destructivo. Hoy en día estamos asistiendo a un cambio de mentalidad y aunque el turismo de masas está lejos de desaparecer, un nuevo tipo de consumidor de productos turísticos está apareciendo. El nuevo turista demanda experiencias únicas, el contacto con la población residente, al mismo tiempo que se muestra preocupado por el bienestar de aquélla y por y preservar el medio natural que lo rodea. El ecoturismo comunitario aparece como un proyecto turístico que combina los distintos aspectos del desarrollo sostenible. Este tipo de turismo se puede adaptar tanto a las comunidades rurales de países en desarrollo como a las comunidades rurales de las zonas periféricas de países desarrollados. Estos proyectos se pueden optimizar si se desarrollan a través de clusters de tamaño pequeño o micro-clusters.

\section{PALABRAS CLAVE}

Desarrollo sostenible - Sostenibilidad económica - Turismo.

\section{ABSTRACT}

While tourism can be an important driver of economic and social development of a destination can also be destructive. Today we are witnessing a change in mentality and even the mass tourism is far from disappearing, a new type of consumer is emerging tourism products. The new tourist demand unique experiences, contact with the resident population, while he was concerned for the welfare of former and preserve the natural environment and surrounding it. Community Ecotourism appears as a tourism project that combines different aspects of sustainable development. This type of tourism can be adapted both to rural communities in developing 
countries and rural communities in outlying areas of developed countries. These projects can be optimized if clusters evolve through small or micro-clusters.

\section{KEY WORDS}

Sustainable development - Economic sustainability - Tourism.

\section{ÍNDICE}

1. Introduction

2. Le nouveau touriste

2.1. Le comportement du nouveau touriste

2.2. L'écoturisme communautaire comme réponse aux attentes du nouveau touriste

2.2.1. Un exemple d'écotourisme communautaire :la réserve indigène des Malekus au Costa Rica

3. Les micro-clusters touristiques

3.1. Les clusters touristiques

3.2. Clusters durables à petite échelle

4. Conclusion

5. Bibliographie

\section{Introduction}

Le tourisme est une industrie qui génère d'importants bénéfices économiques, elle représente d'ailleurs $12 \%$ du PIB mondial. En effet, le tourisme peut offrir des bénéfices économiques et sociaux substantiels à des communautés d'accueil qui ont des opportunités de développement limitées. Toutefois, il est aussi responsable de 
5\% des émissions de $\mathrm{CO} 2$ : la production touristique contribue donc à dégrader les ressources sur lesquelles elle est basée.

Si le tourisme peut être un important moteur du développement économique et social d'une destination, il peut aussi s'avérer destructeur. En effet, le tourisme de masse détruit les ressources naturelles mais aussi parfois l'identité culturelle des destinations.

Or aujourd'hui on assiste à un changement des mentalités et même si le tourisme de masse est loin de disparaître, un nouveau type de consommateur de produits touristiques fait son apparition.

Celui-ci est de plus en plus exigeant sur la qualité de son lieu de vacance et sur l'accueil qui lui est réservé mais il est aussi plus sensible aux valeurs et aux identités culturelles des endroits qu'il visite.

Aujourd'hui, on estime donc que le tourisme doit être «rentable sur le plan économique, durable sur le plan environnemental et équitable sur le plan social» (WWF, 2001).

Le comportement du touriste change et les modalités de mise en place de projets touristiques doivent aussi changer : généralement, les décisions concernant l'activité touristique dans certaines communautés sont prises par les professionnels de l'industrie du tourisme et les gouvernements, les populations locales n'étant que l'objet du projet touristique qu'elles subissent. Cela a très souvent conduit à la détérioration et à l'abandon de nombreuses destinations, laissant la population restée sur place dans de mauvaises conditions, surtout dans les zones rurales. 
Or, une grande part du produit touristique est offerte par ces zones rurales car ces petites localités sont souvent les seules à pouvoir fournir les attributs que recherchent les touristes.

Parallèlement, l'analyse en termes de clusters se développe dans l'industrie du tourisme.

Les clusters sont des formes d'organisation d'industries situées sur un territoire géographique initialement délimité et qui ont été conduites à tisser des relations de nature marchande et non marchande créant ainsi une interdépendance durable tout en conservant leur autonomie. Les clusters sont des réseaux territorialisés qui peuvent se définir

«comme des ensembles coordonnés d'acteurs hétérogènes, géographiquement proches, qui coopèrent et participent collectivement à un processus de production» (Ehlinger et al., 2007).

Les clusters permettent des économies d'échelle qui proviennent d'effets externes dispensés par le milieu économique où se situent les entreprises, de plus, la proximité crée des relations étroites qui améliorent la productivité (Salvador et Chorincas, 2006).

Ainsi, les clusters touristiques, regroupant d'organisations de l'industrie du tourisme et des services associées, permettent d'améliorer la croissance et la performance des destinations grâce aux effets multiplicateurs qui fournissent des bénéfices économiques et qui assurent des retombées en termes de bénéfices sociaux.

Pourtant, très peu d'attention a été portée à la définition et aux effets des clusters de petites tailles. Ces derniers s'avèrent pertinents dans la mise en place de projets touristiques durables au sein de communautés restreintes car ils peuvent être dirigés 
par des choix faits au niveau local afin d'optimiser les conséquences de cette croissance plutôt que de les subir.

Le micro-clustering peut alors permettre de développer de nouvelles destinations touristiques ou aider à l'expansion de destinations existantes tout en faisant coïncider la création de bénéfices économiques avec les besoins et les valeurs de la population locale (Michael, 2007).

Le but de cet article est, dans un premier temps de dresser un portrait du «nouveau touriste» puis d'expliquer comment les micro-clusters touristiques peuvent être une réponse aux attentes de ces derniers tout en permettant une gestion intégrée des ressources naturelles et culturelles.

\section{Le nouveau touriste}

Si les comportements éco-responsables sont difficiles à appréhender (Giannelloni, 1998), il n'en reste pas moins que le comportement du touriste en général a changé. Une lente prise de conscience des dégâts environnementaux et sociaux du tourisme a eu lieu et les touristes souhaitent dorénavant s'engager dans des formes de tourismes plus durables, plus responsables. Nous allons dresser ici un portrait général du nouveau touriste, c'est-à-dire définir ses attentes et ses caractéristiques pour ensuite montrer comment les micro-clusters représentent une réponse adaptée à ce qu'il attend désormais d'un produit touristique.

\subsection{Le comportement du nouveau touriste}

Le tourisme est un phénomène dynamique qui subit les aléas du comportement humain, en ce sens il ne peut être dissocié des changements et des transformations de la société (Le Roy, 1999). Pour appréhender l'évolution du tourisme, il convient d'étudier le comportement du touriste et de montrer que l'on est passé d'une demande touristique de masse, insensible aux effets économiques, 
environnementaux et sociaux du tourisme à une demande plus durable, plus consciente de l'impact des activités touristiques (Fiorello et Bo, 2008).

Le caractère exceptionnel des voyages, l'implication, les risques perçus, la complexité du produit touristique font qu'il est difficile d'appréhender le comportement du touriste. Le statut de touriste est un statut particulier, en effet,

«l'individu acquiert temporairement le rôle de «touriste» en satisfaisant ses besoins récréatifs (relaxation, repos, visite, distractions) et en participant à des activités de transports, d'hébergement, de restauration et de vacances» (Casarin, 2002, p.16).

Avant toute chose, il convient de noter que la demande touristique est loin d'être hétérogène. En effet, il existe différentes typologies du touriste, une seule et même personne pouvant être à la fois plusieurs types de touristes (Meyer, 2005). Néanmoins, il se dégage de grandes tendances permettant de dresser un portrait général du nouveau touriste.

A ces débuts, le tourisme était réservé à une élite, puis, durant les années 1950, la consommation touristique s'élargit à de larges couches de la société et se développe sur un terrain favorable, les «Trente Glorieuses»: croissance économique, allongement de la durée des congés, augmentation du pouvoir d'achat, transformation des modes de vie et une urbanisation croissante qui renforce le besoin de détente (Le Roy, 1999). À cette augmentation de la demande répond une «forme d'organisation de voyage dans laquelle le client achète un «produit» fini et identique à celui qu'achètent les autres clients» (Cuvelier, 1994, p.40).

Essentiellement centrés sur les couples soleil/mer et soleil/montagne, les produits touristiques sont hautement standardisés et aboutissent au développement de 
destinations telles que les Baléares ou la Côte d'Azur. Ce type de tourisme, qualifié de « fordiste » met en scène un touriste inexpérimenté, standard, à la recherche de climats chauds et de soleil dans le cadre de voyages organisés (Poon, 1993). Le tourisme de masse connaît son apogée dans les années 1970-1980 et atteint ses limites quantitatives et qualitatives à la fin du $20^{\mathrm{e}}$ siècle (Moutinho, 2000).

On assiste alors à l'avènement du «nouveau touriste» ou touriste "post-fordiste» (Urry, 1995), qui rejette le tourisme de masse et recherche des produits touristiques différents.

Ce dernier se distingue de l'ancien touriste car il est plus expérimenté, plus soucieux de l'environnement mais aussi plus spontané, plus versatile et plus imprévisible. Habitué à un grand degré de flexibilité et d'indépendance, il recherche des formes alternatives de tourisme (Poon, 1993; Ioannides et Debbage, 1997).

Le modèle de comportement général a donc changé: les voyageurs actuels partent plus fréquemment et pour des périodes plus courtes, les destinations éloignées sont préférées, les individus ne sont plus attachés à une localité particulière et les voyages organisés sont rejetés en faveur de voyages individualisés (Poon, 1993; Urry, 1995). Les motivations pour voyager sont plus variées avec une tendance à la

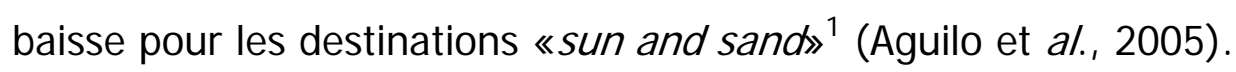

"Ces nouveaux consommateurs veulent se démarquer de la masse. I/s veulent affirmer leur individualité et veulent avoir le contrôle » (Poon, 1993, p. 219).

L'une des caractéristiques essentielles de ce nouveau touriste est son engagement dans une façon responsable de voyager qui se traduit par un souci de respect de l'environnement mais aussi de solidarité envers les populations des pays visités.

\footnotetext{
${ }^{1}$ Destinations soleil/sable (Traduction de l'auteur).
} 
Les spécificités de ce nouveau touriste se retrouvent dans différentes études qui ont cherché a effectué des typologies du touriste. Dans la typologie de King et Hyde (1989) on note la présence des «anti-touristes» qui se perçoivent comme anticonformistes et qui tentent d'être indépendants des autres. Ils évitent le tourisme de masse et accordent une grande valeur à l'authenticité.

Chez Dalen (1989), deux types de touristes se rapprochent de notre portrait du nouveau touriste : les «idéalistes modernes» et les «idéalistes traditionne/s».

Les «idéalistes modernes» recherchent l'excitation et le divertissement mais sont demandeurs d'activités plus intellectuelles, plus culturelles. Ils refusent le tourisme de masse et les itinéraires fixés à l'avance. Les «idéalistes traditionnels» attendent une offre touristique de qualité qui leur assure tranquillité et sécurité mais ils souhaitent également visiter des lieux célèbres et avoir accès à la culture et à la vie locale.

Notre nouveau touriste semble également être la synthèse de trois types de touristes mis en avant par Cohen (1972): le «touriste expérientiel» à la recherche d'expériences authentiques, le «touriste expérimental» dont le désir principal est d'être en contact avec la population et le «touriste existentiel» qui souhaite une immersion totale dans la culture et le style de vie locale.

Enfin, autre type de touriste se rapprochant du «nouveau touriste», le touriste «wanderlust» de Gray (1970) qui souhaite des vacances multi-destinations et qui veut voir et expérimenter des choses uniques, différentes.

Des études plus récentes (Crouch et al., 2005 ; Dolnicar et al., 2008) ce sont, quant à elles, concentrées sur la sensibilité au respect de l'environnement et ont permis de dégager les traits principaux des «environment-friendly-tourists»².

\footnotetext{
${ }^{2}$ Touristes soucieux de l'environnement (traduction de l'auteur).
} 
Les «environment-friendly tourists» se différencient des autres touristes par leurs caractéristiques sociodémographiques, leurs comportements et leurs motivations de voyage (Crouch et al., 2005). Ils sont plutôt jeunes, possèdent un niveau d'éducation assez élevé et un haut niveau de revenu (Dolnicar et al., 2008). Ils se déplacent en famille ou en bande de copains et recherchent la convivialité et les rencontres. De manière générale, ils apprécient l'exercice physique (comme la randonnée), font attention à leur alimentation et aiment la nature (Agridoc, 2004).

Ils souhaitent également enrichir leur connaissance et sont intéressés par le fait d'apprendre des choses lors de leur voyage (Dolnicar et al., 2008).

Mais, le touriste «environment-friendly» n'est pas seulement soucieux de protéger l'environnement car il porte aussi une attention particulière au respect de la communauté d'accueil et à son développement économique et social. II existe donc une attente de la part des touristes pour un tourisme durable qui prendrait en compte tous les aspects du développement durable.

Le «nouveau touriste» est attiré par le contact authentique avec d'autres modes de vie ayant sauvegardé des traditions tout en étant capable d'offrir confort et distractions (Le Roy, 1999). Il est à la recherche d'un dépaysement qui passe autant par le changement de paysage que par la découverte d'éléments culturels et de civilisation. En effet, ce voyageur ne veut pas se limiter à des contacts commerciaux. Il est ouvert aux autres, il est actif et s'intéresse aux populations locales, à leur mode de vie, à leurs coutumes, mais aussi à leurs difficultés. II apprécie les produits du terroir et les recettes locales et est prêt à donner de l'argent pour des projets de développement sous réserve de savoir ce que deviendra cet argent (Agridoc, 2004). II est donc respectueux de ces populations et de leur environnement et attend des résidents un accueil chaleureux et amical et espère que cette hospitalité le mènera à partager leur «intimité». 
Enfin, le nouveau touriste, à la recherche d'authenticité et de la découverte de nouvelles cultures, se tourne de plus en plus vers les espaces ruraux, souvent derniers endroits où la culture locale et les paysages restent préservés.

C'est ainsi, qu'en réponse aux effets pervers du tourisme de masse et aux attentes du «nouveau touriste», de nouvelles formes de tourismes «alternatifs» sont apparues (écotourisme, Pro-Poor Tourism, tourisme solidaire, etc.) Parmi toutes ces nouvelles formes de tourisme, le Community Tourism et plus précisément le Community-Based Ecotourism ou écotourisme communautaire (WWF, 2001) semble être la forme la plus aboutie de tourisme durable en se proposant de

«répondre à la demande sociétale d'une pratique du tourisme respectueuse et qui apporte de la valeur ajoutée économique, sociale et éthique» (Mazuel, 2003, p.333).

\subsection{L'écotourisme communautaire comme réponse aux attentes du nouveau touriste}

Le nouveau touriste, sensible à la culture locale porte donc une attention particulière à l'attitude de la communauté d'accueil. Le comportement de celle-ci devient donc un atout majeur de la destination touristique.

Les individus formant les communautés d'accueil craignent quant à eux de perdre le contrôle sur leur environnement, de perdre leur identité et se montrent souvent réticents à certains projets de développement touristique (Mason et Cheyne, 2000; Capenerhurst, 1994).

L'écotourisme communautaire apparaît comme un moyen de répondre aux nouvelles attentes du touriste et de dépasser les craintes de la communauté d'accueil et ainsi éviter que celle-ci ne se montre hostile envers les touristes en l'impliquant et en la faisant participer à toutes les étapes de l'initiative touristique. 
C'est une forme de tourisme, qui s'adapte plus particulièrement aux espaces ruraux, et basé sur la préservation des ressources naturelles, le bien-être humain et la viabilité économique à long terme des communautés d'accueil (Van der Yeught, 2007), ces dernières devant être, impliquées ou, de préférence, être à l'origine du développement et de l'exploitation des activités touristiques. Elles doivent contrôler de manière significative le développement et la gestion de l'activité touristique et ainsi assurer qu'une proportion importante des bénéfices restera au sein de la communauté.

Getz et Jamal (1994) parlent de «symbiose entre l'environnement et la communauté», Inskeep (1991) appelle à une implication maximum de la communauté locale et Brent Ritchie (1993) soutient que l'attitude des résidents à l'égard du tourisme est l'élément clé de la réussite d'une offre touristique.

Selon Murphy (1985) un engagement total des communautés locales peut permettre de contrôler le rythme du développement touristique, d'intégrer le tourisme dans l'économie et de proposer un produit touristique plus individualisé. Cela nécessite également que la communauté partage une vision de l'offre touristique, cette dernière devant être basée sur la culture et les traditions locales. Les résidents euxmêmes sont donc une part du produit touristique, voire l'élément le plus important.

\subsubsection{Un exemple d'écotourisme communautaire: la réserve indigène des Malekus au Costa Rica}

La réserve indigène des Maleku se situe au nord du pays. Parmi les 24 réserves indigènes que compte le pays, seules 5 d'entre elles, dont la communauté Maleku, ont conservé leurs us et coutumes.

Il y a une dizaine d'année, un chef de la communauté Wilson Morera Elizondo a souhaité sauvegarder la culture maleku et encouragea les jeunes à perpétuer leur 
langue et leurs traditions en leur expliquant que cela permettrait d'apporter une nouvelle source de revenu et de meilleures conditions de vie pour la communauté.

Dans une optique de projet touristique écologique et culturel, il fit construire des ranchos (maisons traditionnelles) avec les matériaux locaux pour accueillir les touristes désireux de découvrir une des premières civilisations du Costa Rica, son environnement naturel, ses traditions et ses connaissances héritées de leurs ancêtres.

Aujourd'hui, ce projet d'écotourisme communautaire permet aux visiteurs de découvrir et de partager le quotidien de la communauté. Les visiteurs sont donc logés dans les ranchs traditionnels et la restauration est constituée de repas typiques, préparés à partir de denrées récoltées dans la réserve.

Les activités proposées sont diverses et essentiellement basées sur la découverte de l'environnement naturel et de la culture maleku: excursions dans les principaux sites d'intérêts écologiques et dans les cultures, découverte de la faune et de la flore, randonnées dans la forêt primaire, participation aux cérémonies indigènes et découverte de la culture des malekus (découverte des plantes médicinales utilisées).

En parallèle sont mis en place un programme de reforestation et un programme de conversation de l'habitat naturel des espèces animales. L'impact écologique du tourisme tend à être minimisé (les déchets sont triés et les déchets organiques donnés aux animaux de la communauté ou transformés en fumier).

Toutes les personnes qui travaillent à ce projet appartiennent à la communauté maleku. Des produits artisanaux confectionnés par les habitants sont vendus dans le centre d'information de la réserve. Enfin, pour chaque touriste, $1 \$$ est reversé dans une caisse commune. (Le petit futé, Guide de l'Écotourisme, 2008) 
L'écotourisme communautaire repose donc sur une réelle prise de pouvoir et d'initiative de la communauté sur l'activité touristique ce que Scheyvens (2002) nomme «empowerment» des communautés et qui passe par un certain niveau de contrôle, de propriété et d'influence (Simpson, 2008).

L'écotourisme communautaire repose sur l'effort de collaboration entre les communautés locales, les intervenants touristiques extérieurs

«et les protecteurs de la ressource, dont l'État, pour préserver les espaces naturels qui servent de support au développement régional». (Lequin, 2001, p.3).

La conduite de projet d'écotourisme communautaire doit reposer sur des principes démocratiques. Les communautés doivent prendre part aux décisions ayant un impact sur la vie de leurs membres, elles doivent pouvoir exercer un contrôle sur les ressources nécessaires pour répondre à leurs besoins et elles doivent être dotées de structures démocratiques et représentatives jouant le rôle d'instances décisionnelles.

Le bon fonctionnement d'un projet d'écotourisme communautaire repose donc sur la bonne gouvernance de celui-ci, c'est-à-dire ne pas imposer de projet touristique à la population locale et veiller à l'intégration de toutes les parties prenantes au projet.

La gouvernance des projets d'écotourisme "repose sur un système ou un réseau d'acteurs dont la logique d'action s'appuie sur la négociation et est axée sur la réalisation d'un produit commun» (Lequin, 2001, p.85), autrement dit, il s'agit de favoriser la confiance interpersonnelle en renforçant la compréhension mutuelle et en stabilisant les attentes.

La communauté d'accueil décide du type et du niveau de tourisme qu'elle souhaite voir se développer, la participation d'entreprises et d'investissements privés extérieur 
n'est pas exclue à condition qu'elle permette à la communauté d'en tirer des avantages et de garder son pouvoir de décision.

A titre d'exemple, en Amazonie, des gîtes ont été construits avec des investissements privés qui offrent une concession à la communauté. C'est-à-dire qu'un accord a été conclu en vertu duquel l'affaire sera remise à la communauté après une période spécifiée pendant laquelle un programme d'emploi et de formation destiné à la population locale sera assuré (WWF, 2008).

Lorsqu'il y a collaboration entre les pouvoirs publics, les entreprises privées extérieures et la communauté locale, celle-ci doit obligatoirement mener à la codécision, à la co-production et au co-management des programmes de développement touristiques.

Toutefois, un certain nombre d'initiatives d'écotourisme n'ont toutefois pas fonctionné «faute d'étude de marché, d'organisation, de qualité du produit et de promotion» (WWF, 2001, p.3).

Cela nous amène donc à nous interroger sur la façon d'appréhender les projets d'écotourisme communautaire et la nécessité de les concevoir à travers la création de clusters de petite taille.

\section{Les micro-clusters touristiques}

Le comportement du touriste a changé, celui-ci ne veux plus que ses activités touristiques aient un impact sur l'environnement ou sur les communautés des destinations qu'il visite. II est également à la recherche de contacts et d'expériences plus authentiques. Ainsi, l'écotourisme qui offre un produit touristique respectueux de l'environnement et des populations est une réponse adéquate à ses attentes, néanmoins pour éviter l'échec de ce type d'initiative il convient de s'interroger sur sa 
mise en œuvre pratique. Notre intuition est que, pour être performant, les projets d'écotourisme doivent être appréhendés sous forme de micro-clusters.

\subsection{Les clusters touristiques}

Selon Porter, une croissance soutenue dépend de la capacité des entreprises à maintenir, sur le long terme, un avantage compétitif, capacité qui est améliorée par la présence de «business units» ou de clusters.

II existe de nombreuses définitions des clusters (Santos et al., 2008)dont nous retiendrons les points essentiels suivants: un cluster est un réseau composé d'entreprises et d'institutions interconnectées, collaborant dans un domaine spécifique et situé dans un espace géographique précis et limité.

Les clusters permettent de générer des emplois, une meilleure compétitivité, des compétences spécialisées, des connaissances nouvelles, des opportunités de coopération, des infrastructures, etc.

La particularité du cluster est que l'entité ainsi créée par différents acteurs à une valeur supérieure à la somme des parties qui la composent.

Le produit touristique, quant à lui, peut être défini comme un ensemble de caractéristiques combinées entre elles: une spécificité géographique et une grande variété de services locaux. Ainsi le tourisme se traduit par l'existence de liens étroits entre des acteurs locaux impliqués dans des activités complémentaires ou concurrentes (Santos et al., 2008).

Les clusters touristiques peuvent alors être définis comme une concentration géographique d'activités culturelles, sociales, environnementales et économiques où des acteurs hétérogènes (entreprises et des institutions) interagissent et coopèrent 
pour augmenter la valeur ajoutée d'un produit et créer un avantage comparatif pour le réseau en question (Dias, 2000 ; Capone, 2004).

C'est un ensemble complexe d'éléments qui incluent les services offerts par les entreprises de l'industrie du tourisme, des infrastructures de transports et de communication, des activités complémentaires et supports, des politiques publiques, des ressources naturelles, des éléments culturels et l'expérience du touriste.

Et, selon Porter (1998), la qualité de cette expérience dépend de l'attrait de la destination (le produit touristique en lui-même) mais surtout de l'existence et de la qualité des activités annexes (restauration, commerces, transport, etc.).

Les clusters touristiques permettent donc à une destination particulière d'être plus compétitive grâce à la coopération et à la mise en commun des ressources entre les différents acteurs présents, ce qui permet de proposer au touriste une offre cohérente.

Enfin, concernant la taille du cluster, son étendue géographique peut varier « $d^{\text {' une }}$ seule ville ou d'une région à un pays entier, voire à un réseau de pays voisins» (Porter, 2004, p. 207).

Or il nous semble que concernant des projets d'écotourisme communautaire, la taille optimale se situe à une micro-échelle.

\subsection{Clusters durables à petite échelle}

Comme nous l'avons vu en présentant l'écotourisme communautaire, la dimension locale, semble être l'échelle optimale pour développer des activités durables. 
Pour soutenir cet argument rappelons qu'une distinction peut être faite entre les rapports qui se nouent dans les groupes sociaux de grande taille et ceux existants dans les groupes de petite taille.

Tönnies (1887) fait une distinction entre société (groupe social de grande voire très grande taille) et communauté (groupe social de taille restreinte). Ainsi, le rapport social peut se fonder sur deux types de volontés: le vouloir naturel, organique, inhérent à l'être et la volonté de choix ou factice qui repose sur la Raison.

C'est le vouloir organique qui porte les conditions de la communauté alors que la volonté réfléchie fonde la société. On comprend ainsi que l'appartenance à une communauté est vécue comme un fait naturel, qui relève de l'évidence.

La communauté est un «organisme vivant» dans lequel les individus sont «organiquement liés» alors que la société est un «agrégat mécanique et artificiel» dans lequel les individus sont "organiquement séparés»: «tandis que dans la communauté, ils restent liés malgré toute séparation, ils sont, dans la société, séparés malgré toute liaison» (Tönnies, 1887 in Chevallier, 1996, p. 103). La confiance, les sentiments et les liens de proximité sont autant de valeur qui fondent une communauté.

Olson (1965), nous dit que la coopération sera plus importante dans de petites communautés stables où les relations sont à une échelle assez faible pour être personnalisées et où les systèmes de comportements sont homogènes. C'est en basant sur cet argument qu' Olson énonce que les biens publics seront plus probablement fournis de manière volontaire dans des petites communautés où les comportements «déviants» (passager clandestin) seront plus facilement repérable et punissable. 
En effet, ce qui différencie les sociétés et les communautés, outre la taille, repose essentiellement sur les types de relations qui se nouent à l'intérieur de ces entités. Les sociétés sont souvent caractérisées par une grande taille et des liens impersonnels. On parle de communauté lorsqu'il s'agit de groupes sociaux de petite taille marqués par une cohésion et une grande coopération entre les membres et où les liens sont étroits et s'apparentent davantage à des liens familiaux ou d'amitié.

L'approche communautaire n'est pas valable dans les groupes, même de petites tailles, marqués par l'individualisme et la compétition.

Schumacher dans son ouvrage "Small is beautifu», publié en 1973, insiste sur la nécessité de créer de petites unités politiques et économiques centrées sur les besoins des personnes et s'attachant à respecter l'environnement, l'égalité et la démocratie.

Le but étant de trouver l'échelle optimale spécifique à chaque activité et d'assurer une cohérence entre le niveau local et une échelle plus globale.

«Du point de vue de l'action, nous avons besoin, de toute évidence, de petites unités, car l'action est une aventure éminemment personnelle, et I'on ne saurait être en relation, à tout moment qu'avec un nombre très restreint de personnes» (Schmacher 1978, Pág. 65).

Envisager l'action à petite échelle facilitera la prise de contrôle par les populations locales et pourra leur permettre d'être à l'origine, du développement d'une activité touristique durable (Murphy, 1985; Getz et Jamal, 1994; Inskeep, 1991; Brent Ritchie, 1993; Mason et Cheyne, 2000).

Nous situons donc le niveau optimal du développement de projet d'écotourisme communautaire à une micro-échelle, celle d'une communauté de petite taille. Pour 
définir la notion de communauté nous reprenons la définition de Doucet et Favreau (1991):

"La communauté se définit par l'existence d'un ensemble de personnes et de groupes disposant de conditions de vie semblables et appartenant à un même espace géographique physique et social. Elle se caractérise également par le partage de valeurs et d'intérêts communs». (Doucet et Favreau, 1991 in Lequin, 2004, Pág.3).

Les clusters écotouristiques doivent prendre la forme de micro-clusters.

L'intérêt accordé aux micro-clusters est récent et repose sur des études réalisées à la fin du $20^{\text {ème }}$ siècle sur une séries de destinations particulières, basées sur l'exploitation d'une niche voire d'une micro-niche de marché: les antiquaires dans le sud-ouest de la Pennsylvanie et en Australie (Grado, Strauss et Lord, 1997; Michael, 2002), les «book towns» en Angleterre et en Europe (Seaton, 1996, 1999). Ces études concernent des régions périphériques et rurales dans des pays développés mais cette approche s'applique également aux pays en voie de développement.

Les micro-clusters se basent donc sur l'exploitation de micro-marchés faisant l'objet d'une demande de la part des touristes.

Seaton (1999) nous montre le succès que connaît la ville de Hay-on-Wye au pays de Galles grâce à son micro-cluster touristique orienté sur le commerce des livres anciens et d'occasion.

Hay-on-Wye, ainsi qu'un certains nombres de zones rurales périphériques européennes ${ }^{3}$, sont devenues des «book towns», autrement dit, des destinations

\footnotetext{
${ }^{3}$ Redu en Belgique, Becherel en Bretagne, Montolieu dans le sud-ouest de la France, Bredevoort aux Pays-Bas, Fjaerland en Norvège et Sysma en Finlande.
} 
touristiques ayant pour particularité de concentrer des commerces vendant des livres d'occasions ou des livres rares.

Avant les années 1960, la ville de Hay n'était pas une attraction touristique et comptait seulement deux hôtels. Aujourd'hui, depuis qu'elle s'est spécialisée dans le commerce du livre, elle est l'une des plus importantes destinations touristique du Pays de Galles qui attire ce que nous avons appelé plus haut des nouveaux touristes ${ }^{4}$. Parallèlement à l'activité «livre», des services se sont développés: restauration, logements et autres loisirs et ce développement s'est fait en assurant un bouleversement minimum de l'environnement naturel, historique et culturel de la ville.

En effet, le développement de ce micro-cluster s'est basé sur la rénovation de bâtiments existants et n'a donc pas nécessité d'entreprendre la construction de nouveaux immeubles. De plus, la multiplication des offres de logement s'est essentiellement fait à travers la création de chambres d'hôtes et de bed and breakfast plutôt que d'hôtels à grande capacité. Les petits commerces ont également été préservés. La communauté locale étant à l'origine de cette initiative, a refusé l'implantation de grandes chaînes (à titre d'exemple, malgré le succès commercial de cette destination, il n'y a pas de McDonald).

Ainsi, les micro-clusters désignent la concentration géographique d'un petit nombre d'entreprises dans un environnement local cohésif (Michael, 2003) où des relations partenariales et coopératives de long terme entre les entreprises et les résidents contribuent à améliorer le niveau de spécialisation locale et à ajouter de la valeur au produit touristique final.

\footnotetext{
${ }^{4} 90 \%$ sont des touristes recherchant la qualité. Ils sont éduqués, appartiennent à une catégorie socioprofessionnelle élevée et ont de hauts revenus (Seaton, 1999, p.391).
} 
C'est une dynamique d'alignement d'activités économiques complémentaires entre des entreprises et des communautés dont l'intention est de proposer un produit touristique spécifique.

En effet, pour endiguer le déclin de certaines zones rurales, des initiatives essentiellement fondées sur le développement de structures d'accueil des touristes ont vu le jour. Mais elles n'ont pas tenu compte des facteurs motivationnels permettant d'attirer des visiteurs (Seaton, 1999). C'est pour cette raison que ces micro-clusters doivent promouvoir un produit unique, facteur clé du succès du marketing d'une destination. Cela permet de communiquer sur une image claire et spécifique et donc de se différencier des autres destinations. Cela ne signifie pas que la zone en question n'offre au touriste qu'un seul type d'activité mais que sa renommée se fonde essentiellement sur ce critère particulier, ce qui améliore la visibilité de la destination.

Selon Seaton (1999), les «book towns » offrent une opportunité de développement touristique soutenable pour les zones rurales périphériques et se caractérisent par les points suivants:

- Ils utilisent des ressources existantes;

- Ils se basent sur la culture, les valeurs et les coutumes locales;

- Ils se développent à petite échelle ce qui donne la possibilité de ne pas effectuer de grosses transformations qui risqueraient de dénaturer le « visage » de la communauté et de détériorer l'environnement;

- Les coûts de départs sont moins importants que pour d'autres initiatives touristiques;

- Les revenus générés restent et sont redistribués au sein de la communauté;

- Ils créent des emplois qui peuvent être pourvus immédiatement; 
- Ils ont des effets multiplicateurs économiques (ils génèrent des activités connexes: restauration, logement, transport et autres services liées à l'industrie du livre);

- Ils sont moins saisonnier et moins dépendant des données climatiques ;

- Ils attirent des touristes internationaux et sont générateurs de nuitées passées sur place (contrairement à certaines destinations qui ne font l'objet que d'une excursion ou d'un « day trip »);

- Ils attirent un nouveau type de touriste (l'antithèse du touriste de masse, plus respectueux);

- Le caractère variable du produit « livre » permet de donner au touriste l'envie d'une deuxième visite ou d'un deuxième séjour.

De manière générale, la démarche de création de micro-clusters doit inclure la planification, le développement, la commercialisation, la gestion des ressources et des services liés à cette forme de tourisme. L'offre se base sur un produit spécifique qui peut être de nature diverse (elle peut concerner des espaces naturels particuliers, un patrimoine culturel, des traditions, etc.) et sera complétée par des services annexes (services de guide et d'interprétation, logement, restauration, vente de produits et d'artisanat, transport, activités de loisirs).

Le micro-cluster ainsi créé peut être un accélérateur de bénéfices économiques et sociaux pour la communauté.

Les micro-clusters touristiques peuvent concourir à un développement régional durable en permettant de préserver et de limiter les impacts sur la nature mais aussi en permettant aux communautés locales de s'engager dans un processus de croissance et de développement dans des conditions acceptables pour eux c'est-àdire compatible avec leurs styles de vie et leurs valeurs. 
Si ces micro-clusters peuvent s'appuyer sur des politiques publiques, cela permettrait de trouver de nouvelles voies pour le développement régional qui resterait en accord avec les attentes des communautés concernées.

Les micro-clusters ont un atout particulier : ils permettent de combiner les avantages des liens de proximité et de confiance qui existent dans les petites communautés et qui facilitent la coopération avec l'existence d'une forme d'organisation qui permet une gestion optimale et performante des activités touristiques.

Ils reposent ainsi sur les deux types de relations sociales énoncées par Weber (1921) : la « communalisation ${ }^{5}$ fondée sur « le sentiment subjectif, traditionnel ou affectif des participants d'appartenir à une même communauté («Zusamengehörigkeit»)»; et la «socialisation» ${ }^{6}$ fondée sur «un compromis d'intérêts motivé rationnellement (en valeur ou en finalité) ou sur une coordination d'intérêts motivée de la même manière» ; (Weber, 1921 in Chevallier, 1996, p.103).

II faut toutefois noter que les micro-clusters, de par leur petite taille, ont un impact limité sur la nature et l'environnement socioculturel mais ont, en contrepartie, un impact limité sur les revenus et sur l'emploi, d'où la nécessité d'intégrer horizontalement et verticalement les micro-clusters.

Cela signifie d'une part de développer l'activité multisectorielle aux seins des communautés (intégration horizontale) et d'autre part d'intégrer ces micro-clusters à d'autres initiatives de même type au niveau local, régional et national.

\section{Conclusion}

\footnotetext{
5 «Vergemeinschaftung»

${ }^{6} \ll$ Vergesellschaftung»
} 
Le nouveau touriste est demandeur d'expériences uniques, de contact avec les populations des pays qu'il visite tout en se montrant soucieux de leur bien-être et de préserver l'environnement naturel qui l'entoure.

L'écotourisme communautaire apparaît ainsi comme un projet de tourisme alternatif qui combine les différents aspects du développement durable. Ce type de tourisme peut s'adapter indifféremment aux communautés rurales de pays en voie de développement qu'aux communautés rurales de zones périphériques dans les pays développés.

De tels projets peuvent être optimisés s'ils sont développés à travers des clusters de petites tailles.

Un micro-cluster touristique est un processus de développement implanté et géré à une échelle «micro». II est basé sur une localité précise et sur l'identification d'un produit unique et permet aux communautés de garder le contrôle de la structure et de la nature de la croissance: ce sont leurs choix et leurs décisions qui vont déterminer la forme du développement et contrôler le management des activités touristiques.

Un micro-cluster touristique performant aura des retombées en termes d'image qui ne se limitent pas à la ville mais qui peuvent concourir au rayonnement du pays.

Si les micro-clusters sont pertinents pour les zones rurales périphériques, il serait intéressant de s'interroger sur la faisabilité d'une initiative de micro-cluster durable au sein de grandes villes.

Enfin, si les évolutions de la demande touristique sont réelles, il est important de garder à l'esprit que le tourisme de masse basé sur le modèle "sun and sand» persiste. En témoigne, entre autres exemples, la continuelle urbanisation à des fins 
touristiques de la Costa Brava en Espagne ou de la «costa Smeralda» au nord de la Sardaigne en Italie.

\section{Bibliographie}

AGRIDOC, (2004): Tourisme solidaire et développement durable. Agridoc, GRET. Paris.

AGUILO, E.; ALEGRE, J. et SARD, M. (2005): "The Persistence of the Sun and Sand Tourism Model" dans la Revue Tourism Management, vol. 26.

BRENT RITCHIE, J., (1993): "Crafting a destination vision - putting the concept of resident-responsive tourism into practice" dans la Revue Tourism Management, 14 (5).

CAPONE, F., (2004): "Regional Competitiveness in Tourist Local Systems" dans le $44^{\text {th }}$ European Congress of the European Regional Science Association (ERSA). Region and fiscal federalism, University of Porto. Porto (Portugal).

CAPENERHURST, J. (1994): "Community Tourism", dans HAYWOOD, L. (Ed.), Community Leisure and Recreation. Butterworth Heinemann. Oxford.

CASARIN, F., ET ANDREANI, J.-C. (2002): "L'étude marketing du comportement du touriste. Problèmes théoriques et enjeux opérationnels" dans la Revue Française du Marketing, vol.188, $n^{\circ} 3$.

CHEVALLIER, J. (1996): Institutions politiques. Librairie Générale de Droit et de Jurisprudence, Paris.

COHEN, E., (1972): "Toward a Sociology of International Tourism" dans la Revue Social Research, 39.

CROUCH, G. ; DEVINNEY, T. ; DOLNICAR, S. ; HUYBERS, T. ; LOUVIERE, J. et CUVELIER, P. (1994) : "Un tourisme post-fordiste?" dans CUVELIER, P., TORRES, E., et GADREY, J.: Patrimoine, modèles de tourisme et développement local. L'Harmattan. Paris.

DALEN, E. (1989): "Research Into Values and Consumer Trends in Norway" dans la Revue Tourism Management, 10 (3). 
DIAS, A., (2000): "Tourism as a Leverage Sector in the New Model of Economic Growth" dans Proceedings of the $12^{\text {th }}$ Meeting $4^{\text {th }}-10^{\text {th }}$ july 1999 . Tourism Sustainability and Territorial Organization. Faro (Portugal).

DOLNICAR, S.; CROUCH, G. I. et LONG, P. (2008): "Environment-Friendly Tourists: What do we Really Know About Them?" dans la Revue Journal of Sustainable Tourism, vol.16, $\mathrm{n}^{\circ} 2$.

ELHINGER, S. ; PERRET, V. et CHABAUD, D. (2007) : "Quelle gouvernance pour les réseaux territorialisés d'organisations?" dans la Revue Française de Gestion, 170.

FIORELLO, A. et BO, D. (2008). "L'émergence du Community Tourism en réponse aux attentes du "nouveau touriste"' dans la Revue Qualitique, $n^{\circ} 202$.

GETZ, D. et J AMAL, T. B. (1994): "The Environment-Community Symbiosis: a Case for Collaborative Tourism Planning" dans la Revue Journal of Sustainable Tourism, 2 (3).

GIANNELLONI, J.-L., (1998): "Les comportements liés à la protection de l'environnement et leurs déterminants: un état des recherches en marketing" dans la Revue Recherche et Applications en Marketing, 13 (2).

GRADO, S.C.; STRAUSS, C.H. et LORD, B.E. (1997): "Antiquing as a tourism recreational activity in Southwestern Pennsylvania" dans la Revue Journal of Travel Research, 35 (3).

GRAY, H.P. (1970): International Tourism: International Trade. Lexington Books. Lexington.

GUIDE DE L'ECOTOURISME, (2008): Le Petit Futé.

IOANNIDES, D. et DEBBAGE, K. (1997): "Post-Fordism and Flexibility: The Travel Industry Polyglot" dans la Revue Tourism Management, vol.18, $n^{\circ} 4$.

INSKEEP, E. (1991): Tourism Planning: An Integrated and Sustainable Development Approach. Van Nostrand Reinhold. New York.

KING, B. et HYDE, G. (1989): Tourism Marketing in Australia. Hospitality Press. Melbourne.

LE ROY, A. (1999): "Tourisme et dynamiques territoriales rurales" dans Les Cahiers de l'Espace, série «Cahiers de l'Espace Europe», n¹5, décembre 1999. 
LEQUIN, M. (2001): Ecotourisme et gouvernance participative. Presses de l'Université du Québec. Québec (Canada).

MASON, P. et CHEYNE, J. (2000): "'Residents' Attitudes to Proposed Tourism Development" dans la Revue Annals of Tourism Research, vol.27, n².

MAZUEL, L. (2003). "Développement durable: I'exemple privilégié du tourisme rural" dans SPINDLER, J. et DURAND, H. : Le Tourisme au XXI siècle. L'Harmattan. Paris.

MEYER, C., (2005) : "Le tourisme: essai de définition" dans la Revue Management et Avenir, 1 (3).

MICHAEL, E. J. (2002): "Antiques and Tourism in Australia" dans la Revue Tourism Management, $23(2)$.

MICHAEL, E. J. (2003): "Tourism micro-clusters" dans la Revue Tourism Economics, $9(2)$.

MICHAEL, E. J. (2007): Micro-clusters and network, the growth of tourism. Elvisier.

MOUTINHO, L. (2000): "Trends in Tourism", dans MOUTINHO, L. (Ed.): Strategic Management in Tourism. CAB International. Wallingford.

MURPHY, P. E. (1985): Tourism: A Community Approach. Methuen. Londres.

OLSON, M. (1965): The logic of collective action: public goods and the theory of group. Harvard University Press. Cambridge, Masachusets.

PORTER, M. (1998): On Competition, Harvard Business School Press. Harvard. Boston.

PORTER, M. (2004): Competitiveness in Rural U.S. Regions. Learning and Research Agenda, Institute for Strategy and Competitiveness. Harvard Business School. Harvard. Boston.

POON, A. (1993): Tourism, Technology and Competitive Strategies. CAB international. Wallingford.

SALVADOR, R. et CHORINCAS, J. (2006): "Les clusters régionaux au Portugal", Géographie, Économie Société, 4 (8).

SANTOS, C.; ALMEIDA, A. et TEIXEIRA, A. A. (2008): Searching for Cluster in Tourism. A Qualitative Methodological Proposal. Working Paper. Université de Porto. Porto (Portugal). 
SCHUMACHER, E. F. (1978): Small is beautiful: une société à la mesure de l'homme, Contretemps: le Seuil. Paris.

SCHEYVENS, R. (2002): Tourism for Development: Empowering Communities, Pearson Education. Harlow.

SEATON, A.V. (1996): "Hay on wye, the mouse that roared, book towns and rural tourism" dans la Revue Tourism Management, 17 (5).

SEATON, A.V. (1996): "Hay on Wye, the Mouse that Roared: Book Towns and Rural Tourism" dans la Revue Tourism Management, 17 (5).

SEATON, A.V. (1999): "Book towns as tourism development in peripheral areas", International Journal of Tourism Research, 1.

SIMPSON, M. C. (2008): "Community Benefit Tourism Initiatives - A Conceptual Oxymoron?" dans la Revue Tourism Management, vol.29.

TÖNNIES, F. (1887): Communauté et société. PUF. 1944. Paris.

URRY, J. (1995): Consuming places. Routledge. Londres.

VAN DER YEUGHT, C. (2007): "La Resource Based View et le développement durable au service de l'analyse stratégique: une application aux destinations touristiques" dans les Actes de la XVIème Conférence de l'Association Internationale de Management Stratégique. Montréal, Canada.

WORLD WILDLIFE FUND, WWF (2001): Lignes directrices pour le développement de l'écotourisme communautaire, WWF International. 